\title{
Transparência, corrupção e má gestão: uma análise dos municípios brasileiros
}

\author{
Mariana Batista 1 \\ Virginia Rocha ${ }^{2}$ \\ José Luiz Alves dos Santos ${ }^{3}$ \\ 1 Universidade Federal de Pernambuco / Departamento de Ciência Política, Recife / PE - Brasil \\ 2 Universidade Federal de Pernambuco / Programa de Pós-Graduação em Ciência Política, Recife / PE - Brasil \\ ${ }^{3}$ Universidade Federal de Pernambuco / Graduação em Ciência Política, Recife / PE - Brasil
}

Compromisso institucional com a transparência pública tem relação negativa com corrupção e má gestão governamental? A instituição de Lei de Acesso à Informação (LAI) reduz o custo de acesso à informação pública e o controle dos governantes sobre ela, possibilitando o monitoramento do governo. Assim, maior transparência melhoraria a performance governamental. Para testar a hipótese, usamos modelos de regressão linear para 320 municípios brasileiros. Empregamos 3 medidas do compromisso institucional dos municípios com a transparência pública: a) a regulamentação da LAI no município; b) o grau de regulamentação com base nos dispositivos da LAI em nível local; e c) o tempo de adesão como medida de institucionalização da transparência pública. Como medida de performance, consideramos irregularidades de corrupção e má gestão identificadas nos relatórios de auditorias da Controladoria-Geral da União via sorteios públicos (2011-2015), com classificação usando aprendizado de máquina não supervisionado. Os resultados da análise são 3: a) a adesão dos municípios à LAI ainda é muito reduzida; b) nos municípios que aderiram, transparência não apresenta associação com a performance governamental; e c) apenas desenvolvimento econômico e qualidade da burocracia apresentam correlação negativa com o número de irregularidades. Os resultados indicam que o esforço na direção de uma gestão mais transparente ainda não gerou os resultados esperados.

Palavras-chave: transparência; lei de acesso à informação; corrupção; má gestão; governo local.

\section{Transparencia, corrupción y mala gestión: un análisis de los municipios brasileños}

¿El compromiso institucional con la transparencia pública reduce la corrupción y la mala gestión del gobierno? La institución de la Ley de Acceso a la Información (LAI) reduce el costo de acceso a la información pública y el control de los funcionarios del gobierno sobre ella, lo que permite que el gobierno sea monitoreado. Por lo tanto, una mayor transparencia mejoraría el desempeño del gobierno. Para probar la hipótesis, usamos modelos de regresión lineal en 320 municipios brasileños. Empleamos tres medidas del compromiso institucional de los municipios con la transparencia pública: la regulación de la LAI en el municipio; el grado de regulación basado en las disposiciones de la LAI a nivel local; y el tiempo de adhesión como medida de institucionalización de la transparencia pública. Como medida de rendimiento, consideramos las irregularidades de corrupción y mala gestión identificadas en los informes de auditoría de la Contraloría General de la Federación a través de sorteos públicos (2011-2015), con clasificación mediante aprendizaje automático no supervisado. Los resultados del análisis son tres: la adhesión de los municipios a la LAI todavía es muy baja; en los municipios que se adhirieron la transparencia no está asociada al desempeño del gobierno; y solo el desarrollo económico y la calidad de la burocracia tienen correlación negativa con el número de irregularidades. Los resultados indican que el esfuerzo hacia una gestión más transparente aún no ha generado los resultados esperados.

Palabras clave: transparencia; ley de acceso a la información; corrupción; mala gestión; gobierno local. 


\section{Transparency, corruption, and mismanagement: an analysis of Brazilian municipalities}

Does institutional commitment to public transparency reduce corruption and public mismanagement? The regulation of freedom of information laws (FOI) reduces the cost of accessing public information and the control of government officials over it, allowing for monitoring of the government. Thus, greater transparency improves government performance. To test the hypothesis, we used linear regression models for 320 Brazilian municipalities. We employ three measures of municipality institutional commitment to public transparency: FOI regulation in the municipality; the degree to which the local level regulation was based on the national law's provisions; and time the municipality took to adhere to the legislation, as a measure of institutionalization of public transparency. As a performance measure, we consider corruption and mismanagement irregularities identified in the audit reports of the Comptroller General of Brazil (2011-2015), with classification using unsupervised machine learning. The results are threefold: the municipalities' adherence to FOI is still very low; in the municipalities that regulated the law locally, transparency is not seen to be associated with government performance; only economic development and quality of bureaucracy have a negative correlation with the number of irregularities. These results indicate that the effort towards more transparent management has not yet generated the expected results.

Keywords: transparency; freedom of information law; corruption; mismanagement; local government.

\section{AGRADECIMENTOS}

O presente trabalho foi desenvolvido no âmbito do projeto "Mais Recursos ou Melhor Gestão" que recebeu financia,mento da Fundação de Amparo à Ciência e Tecnologia do Estado de Pernambuco (FACEPE), processo: APQ-0158-7.09/15. José Luiz Alves dos Santos agradece o recebimento de bolsa de iniciação científica pela FACEPE para o desenvolvimento da presente pesquisa e Virgínia Rocha o recebimento de bolsa de doutorado da CAPES.

\section{INTRODUÇÃO}

O compromisso institucional com transparência pública diminui a corrupção e a má gestão governamental? Existe uma expectativa teórica e prática de que transparência aumente a participação, reduza a corrupção e melhore a performance do governo (Calland \& Bentley, 2013; Cucciniello, Grimmelikhuijsen, \& Porumbescu, 2016; Fox, 2007; Meijer, Curtin, \& Hillebrandt, 2012; Prat, 2005). Com isso, tal fenômeno tem atraído a atenção de estudiosos, pautado a ação de advocates do acesso à informação e justificado reformas e iniciativas internacionais (Bauhr \& Grimes, 2012; Calland \& Bentley, 2013; Fox, 2007). Neste artigo, abordamos a regulamentação da Lei n. 12.527 (Lei de Acesso à Informação [LAI], 2011) nos municípios brasileiros e, portanto, focamos em um tipo específico de transparência: o regime de liberdade de informação (Heald, 2006; Kosack \& Fung, 2014). Enquanto 119 países já adotaram algum tipo de regime de liberdade de informação (Freedom of Information [FOI], 2019), os efeitos dessa política são mistos e ainda precisamos avançar muito na avaliação de seus impactos (Calland \& Bentley, 2013; Cucciniello et al., 2016; Michener, 2019).

No Brasil, o escopo da LAI (2011) é nacional e seus efeitos imediatos valem para todos os órgãos da administração pública, seja em nível federal, estadual ou municipal. Contudo, a regulamentação da LAI (2011) depende de ação autônoma dos estados e municípios, estabelecendo regras de acesso, instrumentos de transparência passiva e ativa e também procedimentos de sigilo. Dessa forma, é possível pensar na regulamentação como a adesão do ente federativo à transparência pública estimulada pela LAI (2011). Com a análise da adesão dos municípios aos procedimentos da transparência, podemos 
identificar sua difusão no território e, mais importante, quais resultados tangíveis a transparência traz para a gestão pública local. Assim, tratamos a regulamentação da LAI (2011) como a formalização do compromisso institucional de um governo com dispositivos legais que visam a garantir a qualidade da informação disponibilizada sobre as ações desenvolvidas pelo governo.

O acesso à informação é constantemente associado ao combate à corrupção e à melhoria da gestão pública. De acordo com o manual da LAI (2011) para estados e municípios (ControladoriaGeral da União [CGU], 2013, p. 6), “o acesso às informações públicas é um requisito importante para a luta contra a corrupção, o aperfeiçoamento da gestão pública, o controle social e a participação popular".

Com isso em mente, o objetivo deste artigo é testar o argumento de que maior compromisso institucional legal com a transparência é associado com a redução da má gestão e da corrupção nos municípios brasileiros. Apesar de intuitivamente parecer óbvia, a relação entre transparência e desempenho da gestão pública não é automática. Há argumentos de que transparência seja insuficiente para reduzir corrupção (Bahur \& Grimes, 2012; Fox, 2007). Diante disso, esse estudo pretende usar uma medida direta de corrupção e má gestão a partir das irregularidades detectadas pela CGU. Usamos um banco de dados original que operacionaliza as irregularidades identificadas pela CGU no Programa de Fiscalização por Sorteios Públicos como má gestão e corrupção.

Diferenciamos corrupção de má gestão com base no ganho direto do gestor. Quando as irregularidades são associadas a fraudes em processos licitatórios, classificamos como corrupção. Quando as irregularidades são relacionadas a atos que não envolvem o ganho do gestor, como desperdício de remédios ou falta de controle de frequência escolar, classificamos como má gestão. Identificamos mais de 16 mil irregularidades nas auditorias realizadas pela CGU entre 2011 e 2015, para um total de 320 municípios. O período de análise se inicia no ano de promulgação da LAI (2011) e se encerra em 2015, último ano no qual a CGU usou a metodologia de sorteios para seleção dos municípios. Essa metodologia foi posteriormente modificada, afetando a continuidade dos dados. Realizamos uma classificação automatizada das irregularidades por meio de um algoritmo de aprendizado de máquina não supervisionado. Usamos 3 medidas do compromisso institucional dos municípios com a LAI (2011) com base em dados do Instituto Brasileiro de Geografia e Estatistica (IBGE): a) a regulamentação da LAI (2011) no município; b) o grau de regulamentação com base nos dispositivos da LAI (2011) em nível local; e c) o tempo de adesão como medida de institucionalização da transparência pública.

Esperamos que um maior compromisso institucional com o acesso à informação a partir da adesão à LAI (2011), em suas 3 medidas, esteja associado com menor número de casos de irregularidades em geral e de corrupção e má gestão especificamente. Testamos nosso argumento central a partir de um desenho de pesquisa quantitativo, usando técnica de regressão linear. Dessa forma, visamos a contribuir para o debate sobre o acesso à informação e a performance governamental ao trazer evidências empíricas inéditas, que tratam sobre os efeitos das políticas que objetivam combater as ações ilícitas presentes na gestão pública.

Para tanto, após esta Introdução, o restante do artigo se organiza assim: a) a primeira seção apresenta o debate teórico sobre a relação entre transparência pública e performance governamental; b) a segunda seção apresenta a metodologia; c) a terceira seção apresenta os resultados alcançados; e d) por último, apresentamos nossa conclusão. 


\section{TRANSPARÊNCIA E PERFORMANCE GOVERNAMENTAL}

A qualidade do governo tem sido tratada como peça-chave nos problemas sociais e econômicos enfrentados pela sociedade (Rothstein \& Teorell, 2008). Ela pode ser compreendida, em linhas gerais, como a performance dos agentes que executam a vontade dos principais (Fukuyama, 2013).

Sob uma visão normativa, a qualidade do governo pode ser entendida como o exercício imparcial do poder público. Isto é, implementar leis e políticas de acordo com aquilo que foi definido por elas ex ante (Rothstein \& Teorell, 2008). Assim, há um pressuposto de que imparcialidade implica capacidade suficiente do Estado. Entretanto, um Estado pode ser, ao mesmo tempo, imparcial e incapaz de prover serviços públicos de forma efetiva (Fukuyama, 2013). Dessa forma, sob uma perspectiva empírica, é importante considerar a interação entre a capacidade, medida pela capacidade extrativa do Estado e qualificação de sua burocracia, e a autonomia dessa burocracia para executar políticas (Fukuyama, 2013; Marenco, 2017; Marenco, Strohschoen, \& Joner, 2017).

Com base nesses argumentos:

- Consideramos performance governamental a capacidade dos governos de implementar políticas e leis conforme critérios por eles estabelecidos previamente.

A violação dessa imparcialidade pode ocorrer tanto por falta de capacidade de executar o que foi planejado quanto pelo objetivo de obter vantagens indevidas a partir da execução parcial do poder. Esses casos indicam, respectivamente, má gestão e corrupção. Má gestão é o comportamento e/ou as decisões que levam à baixa performance, o que difere de infortúnio, isto é, de situações adversas que fogem do controle da organização e afetam seu desempenho (Andrews, Boyne, \& Enticott, 2006). Já corrupção seria a venda de propriedade do governo para obter vantagens pessoais (Shleifer \& Vishny, 1993). Tais vantagens podem ser ganhos eleitorais ou materiais (Nyblade \& Reed, 2008). Assim, corrupção se refere à transação entre um político e um ator privado, de um bem público ou direito de propriedade para obter ganhos materiais ou eleitorais (Batista, 2013). Essa transação ocorre de uma forma que contraria as regras do jogo (Jain, 2002).

Diante disso, observamos as irregularidades detectadas pela CGU por meio das auditorias do Programa de Fiscalização por Sorteios Públicos, que verifica a correta aplicação de recursos federais repassados a entes federativos. Diversos estudos têm usado esses dados especialmente como proxy de corrupção, seja para identificar determinantes da corrupção em nível local (Albuquerque \& Ramos, 2006); observar a relação entre emendas parlamentares ao orçamento e corrupção municipal (Sodré \& Alves, 2010); examinar como reeleição afeta corrupção (Ferraz \& Finan, 2011); ou, ainda, verificar como competição eleitoral afeta a corrupção (Batista, 2013).

Um argumento subjacente a esses estudos é o problema da delegação da tomada de decisão e da implementação de políticas do modelo de principal-agente. Nesse sentido, o controle do eleitor sobre o governante depende de 2 fatores: a) eleições regulares e livres, isto é, a possibilidade de perder o mandato; e b) o grau de informação dos eleitores, o qual afeta as oportunidades dos governantes para se engajar em comportamento desonesto (Adserà, Boix, \& Payne, 2003). Enquanto o modelo de Barro (1973) prevê informação perfeita, Ferejohn (1986) insere o pressuposto de informação assimétrica - os eleitores não podem observar diretamente o comportamento do governante. Este, por sua vez, 
beneficia-se dessa assimetria - quanto maior sua vantagem informacional, maior sua capacidade de extrair renda a partir de seu mandato.

Esse debate influenciou análises que investigam como variáveis da dimensão eleitoral, como competição e reeleição, disciplinam governantes (Batista, 2013; Chang, 2005; Chang \& Golden, 2007; Ferraz \& Finan, 2011; Pereira, Melo, \& Figueiredo, 2008; Persson, Tabellini, \& Trebbi, 2003), bem como pautou estudos que passaram a examinar como mecanismos de transparência e participação afetam a performance do governo, especialmente após iniciativas como a Parceria pelo Governo Aberto (Fukuyama, 2016). Por outro lado, a rápida disponibilização de informação pela internet também gerou externalidades negativas, a exemplo da instabilidade de investimentos, o que lança luz sobre os efeitos não intencionais da transparência (Fukuyama, 2016).

Evidências da relação positiva entre informação e melhoria da performance governamental (Adserà et al., 2003; Cucciniello et al., 2016; Fukuyama, 2016; Lindstedt \& Naurin, 2010), contrastam com evidências nulas ou negativas, as quais indicam que o acesso à informação não é suficiente para alcançar os resultados esperados (Bataglia, 2019; Bauhr \& Grimes, 2012; Casalecchi \& Oliveira, 2010; Costa, 2012; Escaleras, Lin, \& Register, 2010; Fukuyama, 2016). Educação, circulação da mídia e eleições livres e justas também são fatores importantes nessa relação (Brunetti \& Weder, 2003; Lindstedt \& Naurin, 2010).

Não obstante, a associação entre transparência e melhora no comportamento dos governantes é antiga. No século XVIII, Jeremy Bentham (1995) desenvolveu um projeto de prisão chamada pan-óptico. Nela, os guardas estavam posicionados no centro da construção, de uma forma em que era possível enxergar todos os prisioneiros (Bentham, 1995). A consciência de serem monitorados o tempo todo seria suficiente para incentivar o bom comportamento (Batista, 2017; Schedler, 1999). Nesse sentido, governantes podem antecipar o monitoramento decorrente da transparência, sem que esse escrutínio necessariamente exista, e evitar comportamentos corruptos, por exemplo (Meijer et al., 2012). Tais ideias, porém, carregam a expectativa de que indivíduos processam informação da mesma maneira, sem considerar a variedade de vieses cognitivos existentes (Rodrigues, 2019).

Para compreender essa multiplicidade de argumentos e evidências, é central observar os diferentes tipos de transparência e a quais resultados eles podem levar. Prat (2005), por exemplo, defende que enquanto a transparência sobre as consequências das ações dos agentes pode ser benéfica, a transparência sobre suas ações em si pode gerar efeitos negativos sobre a disciplina e seleção dos agentes, sendo maléfica para o principal. Michener e Bersch (2013) definem transparência de acordo com seus elementos constitutivos: visibilidade e inferabilidade. A primeira está associada ao grau no qual a informação é completa $e$ facilmente encontrada. Já a segunda está ligada à qualidade da informação e ocorre quando esta é desagregada ou simplificada ou verificável (Michener \& Bersch, 2013).

Por sua vez, Heald (2006) apresenta quatro direções da transparência que variam quanto ao que pode ser observado (externa ou internamente) e em qual sentido - vertical ou horizontal (Rodrigues, 2019). A direção vertical e ascendente permite que o superior na hierarquia observe seus subordinados, sendo baseada no modelo econômico do principal-agente. Já na descendente, os governados podem observar seus governantes, o que remete à accountability è democracia. No eixo horizontal, a transparência pode ser voltada para o exterior, quando o agente pode observar o que acontece fora da organização, ou para o interior, quando é possível observar o que ocorre dentro de uma organização. Este último tipo de direção de transparência está ligado ao regime de liberdade de 
informação (freedom of information [FOI]), além de mecanismos de controle social. Tal direção diz respeito à vigilância e ao monitoramento entre pares (Heald, 2006). A liberdade de informação seria usada por jornalistas e cidadãos com o objetivo de que os indivíduos possam governar-se melhor democraticamente (Kosack \& Fung, 2014).

A partir dessas direções, Heald (2006) deriva 3 dicotomias que representam variedades da transparência, sendo as 2 primeiras o momento no qual a informação é divulgada (transparência em retrospecto $\times$ transparência em tempo real); e se a informação se refere a um evento ou ao processo em si. A terceira dicotomia é entre transparência efetiva e transparência nominal. O gap entre ambas indica uma ilusão da transparência, a qual pode ocorrer quando o governo não disponibiliza informação da maneira adequada ou quando o receptor não possui capacidade cognitiva para interpretar e usar aquela informação.

Rodrigues (2019) argumenta que o movimento da transparência nominal para uma transparência plena demonstra esforço das instituições para superar as falhas que impedem o exercício pleno da transparência. Dado que uma transparência plena, na qual há tanto visibilidade quanto inferabilidade da informação, é muitas vezes inviável na gestão pública, por conta da necessidade de sigilo, a autora avalia o grau de visibilidade e inferabilidade possível nos diferentes tipos e variedades de transparência destacados por Heald (2006). Assim, na transparência nominal, a inferabilidade é limitada, mas a visibilidade está presente. Ou seja, a informação é acessível, mas não permite que inferências sejam feitas a partir dela.

Ao abordarmos a LAI (2011) neste estudo, analisamos a transparência como a formalização do compromisso institucional de um governo com dispositivos legais que visam garantir a visibilidade e a inferabilidade da informação disponibilizada pelo governo sobre as ações desenvolvidas por essa organização. Não há, nesse caso, garantia de que a transparência será efetiva, mas sim a sinalização de um esforço institucional para que isso aconteça. Dessa forma, embora a liberdade de informação se relacione tanto com as direções vertical descendente e horizontal voltada para dentro (Heald, 2006), não estamos focados em iniciativas que visam ao fomento da accountability, as quais têm direção vertical descendente (Heald, 2006) e usuários que usam serviços específicos (Kosack \& Fung, 2014), mas sim em seu aspecto horizontal. Isto é, nos instrumentos adotados por governos municipais via LAI (2011) na expectativa de que as informações fornecidas sobre o governo sejam de qualidade, contemplando os aspectos de visibilidade e inferabilidade discutidos por Michener e Bersch (2013).

As leis de liberdade de informação oferecem arcabouço legal para o acesso à informação interna do governo, representando uma medida institucional de transparência (Hollyer, Rosendorff, \& Vreeland, 2014), ao definir regras e procedimentos para sua aplicação, bem como reduzir o controle dos representantes sobre a informação e permitir que ela possa ser usada para monitoramento do governo (Berliner, 2014). Tal institucionalização torna mais custoso para os governantes retroceder à cultura do silêncio (Berliner, 2014). Ademais, mesmo quando governantes regulamentam a FOI apenas para obter benefícios eleitorais, com o tempo, a atenção da opinião pública pode mover-se da adoção para a implementação da lei, o que aumenta os custos reputacionais de retrocesso à cultura do sigilo (Schnell, 2017).

Almejamos entender se a variação no compromisso institucional legal que os governos têm com o acesso à informação, observado por meio da regulamentação da LAI (2011) em três medidas distintas, está associada com menor incidência de irregularidades relacionadas à corrupção e à má gestão no nível local. Argumentamos que esse compromisso está associado com a redução de 
má gestão e corrupção e melhor a performance do governo. A ideia é que municípios que regulamentaram a LAI (2011), especialmente em um maior grau de regulamentação e há mais tempo, têm maior comprometimento com o acesso à informação, cultura do silêncio menos enraizada, e transparência mais institucionalizada. Assim, propomos as seguintes hipóteses:

1) Municípios que regulamentaram a LAI apresentam menor quantidade de irregularidades relacionadas à má gestão e à corrupção;

2) Quanto maior o grau de regulamentação da LAI, menor a quantidade de irregularidades relacionadas à má gestão e à corrupção nos municípios brasileiros;

3) Quanto maior a institucionalização da LAI, menor a quantidade de irregularidades relacionadas à má gestão e à corrupção nos municípios brasileiros.

Além do acesso à informação, também consideramos explicações alternativas para a performance governamental, as quais foram muito brevemente discutidas nesta seção por uma limitação de espaço. Assim, incluímos como controles nesta análise: a) incentivos eleitorais (reeleição); b) qualidade da burocracia local; c) meios de comunicação; d) desenvolvimento econômico; e e) região ${ }^{1}$. Esperamos que prefeitos em primeiro mandato estejam associados a menos irregularidades, já que os incentivos eleitorais podem restringir o comportamento desviante. Quanto à burocracia, que é responsável pela implementação dos programas analisados, esperamos que quanto maior a qualidade da burocracia, menor o número de irregularidades na gestão local. Testamos se a presença de meios de comunicação melhora a performance, uma vez que esses são veículos que aumentam o alcance da informação pública. Por fim, esperamos que desenvolvimento econômico e região controlem as diferenças estruturais entre os municípios no Brasil.

\section{DADOS E MÉTODOS}

Para identificar a relação entre transparência e performance governamental, analisamos os municípios brasileiros no período de 2011 a 2015. Adotamos o ano de promulgação da LAI (2011) como ponto de partida para a análise. $\mathrm{O}$ ano de 2015 é o ponto final devido à disponibilidade de dados sobre a performance governamental, como descrito abaixo. O nível municipal foi escolhido porque mantém fixas as instituições do país, ao mesmo tempo que oferece variabilidade tanto no tocante à adoção da LAI (2011) quanto ao contexto político e socioeconômico.

Todos os dados sobre a regulamentação da LAI (2011) no Brasil são da pesquisa Perfil dos Municípios, conduzida pelo IBGE no ano de 2014. Essa edição da pesquisa contém um módulo especial sobre transparência e acesso à informação. Nessa pesquisa há informações sobre a regulamentação da LAI (2011), os dispositivos presentes e também o ano de adoção, que nos permite identificar há quanto tempo o município tem a LAI (2011) em funcionamento - dados obtidos em IBGE (2020).

Como medida de regulamentação da LAI (2011), usamos a variável que indica se o município regulamentou a LAI (2011) ou não. Trata-se, portanto, de uma variável binária que assume o valor 1 quando há regulamentação e valor 0 quando não. A institucionalização da LAI (2011) é uma medida

\footnotetext{
${ }^{1}$ Sobre os efeitos dos incentivos eleitorais da reeleição, ver Pereira, Melo, e Figueiredo (2008) e Ferraz e Finan (2011). Sobre o efeito da capacidade burocrática local, ver Batista (2015). E sobre o efeito dos meios da comunicação, ver Ferraz e Finan (2011).
} 
que conta o número de anos de implementação da LAI (2011) no município. Essa variável assume o valor máximo de 4, para os municípios que regulamentaram a LAI (2011), e valor mínimo de 0, para aqueles municípios que ainda não regulamentaram a LAI (2011).

O grau de regulamentação é um índice composto pelos dispositivos previstos pela LAI (2011) na regulamentação local. Usamos 8 indicadores. Todas as variáveis assumem valor 0 quando ausente e 1 quando presente na regra local. A variável final é a soma dos indicadores e, portanto, tem valor mínimo de 0 e máximo de 8. Os indicadores são os seguintes: a) Estabelece prazo de atendimento; b) Institui procedimentos de classificação de informação; c) Prevê autoridade de monitoramento; d) Prevê órgão central de monitoramento; e) Prevê a divulgação de relatório de monitoramento; f) Prevê mais de uma instância recursal interna ao órgão ou entidade demandado(a); g) Estabelece instância recursal autônoma e independente em relação à autoridade que proferiu a decisão impugnada; h) Estabelece procedimento em caso de omissão de resposta.

Para mensurar a performance do governo local, usamos três medidas com base nos dados das auditorias da CGU. Esses dados são informações objetivas sobre a gestão de recursos federais em nível municipal. Com a municipalização de algumas das principais políticas sociais, o nível municipal se tornou central na gestão de recursos públicos e na entrega de benefícios e serviços primordiais à população. Por essa razão, os dados de auditoria da CGU são simultaneamente relevantes no objeto que avaliam e apropriados para um teste do efeito da transparência, já que se referem à gestão dos recursos e não a seus impactos na sociedade, algo que é afetado por inúmeros outros fatores.

Além de ser uma medida objetiva, os dados são gerados aleatoriamente, seguindo o sistema de sorteio de loterias da Caixa Econômica Federal. Aqui, usamos os relatórios produzidos no período de 2011 a 2015, ano em que o programa passou por reformulações e deixou de ser aleatório. Nesse período, 320 municípios foram auditados. O desenho das auditorias da CGU prevê que somente municípios de pequeno porte (população de 10.000 até 250.000 habitantes) são auditados. Por essa razão, a amostra, apesar de aleatória, não é representativa da população de municípios no Brasil. Mesmo assim, acreditamos que o estudo oferece subsídios para entendermos as implicações da transparência sobre o desempenho da gestão local.

Os relatórios de auditoria da CGU são arquivos de texto onde as irregularidades encontradas são descritas. Esses relatórios podem ser bastante detalhados, chegando a arquivos de 300 páginas. Para esta pesquisa, precisamos apenas da descrição básica da irregularidade. Para tanto, extraímos manualmente a síntese das irregularidades apresentadas nos relatórios ${ }^{2}$. Esse primeiro procedimento gerou um arquivo com 16.064 irregularidades.

O próximo passo foi classificar o tipo de irregularidade. Para evitar subjetividade na classificação, usamos um algoritmo de aprendizado de máquina não supervisionado chamado Latent Dirichlet Allocation (LDA). Esse é um procedimento probabilístico que consegue recuperar automaticamente as palavras que compõem o tópico e os tópicos que compõem o documento (Blei, 2012). Para nossa aplicação, o resumo da irregularidade é usado como documento e, a partir da implementação do procedimento, é possível estimar a probabilidade de uma palavra compor o documento $(\beta)$ e a distribuição de tópicos no documento $(\delta)$. O LDA é um algoritmo de classificação em tópicos (topic

\footnotetext{
${ }^{2}$ Procedimentos automatizados não foram bem-sucedidos em recuperar corretamente o número de irregularidades presentes em um relatório.
} 
classification) que recupera múltiplos tópicos. Para nossos objetivos, somente o tópico com maior proporção no texto é usado.

Recuperamos 4 tópicos a partir das palavras observadas nas irregularidades. Os rótulos dos tópicos são de nossa responsabilidade. Nomeamos os tópicos da seguinte forma: a) licitação; b) gastos; c) execução; e d) utilização. A Figura 1 apresenta as palavras associadas a cada tópico. Para validar a classificação, uma amostra de 160 irregularidades foi classificada manualmente, com taxa de acerto superior a $90 \%$, indicando a validade do procedimento.

\section{FIGURA 1 TÓPICOS E PALAVRAS EXTRAÍDOS DAS EMENTAS DAS IRREGULARIDADES}
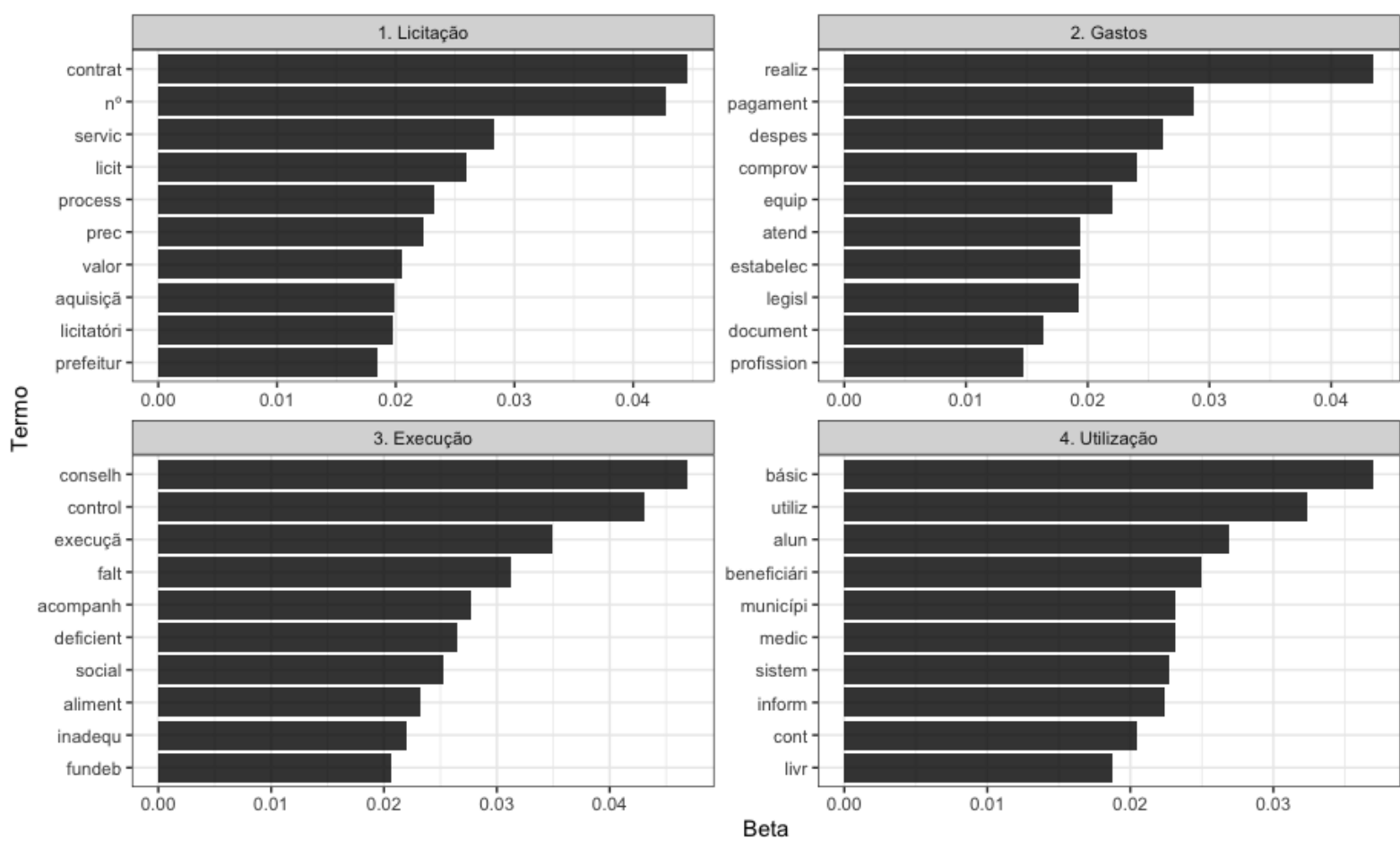

Fonte: Elaborada pelos autores.

Uma vez identificados os tópicos aos quais as irregularidades se referem, dividimos as irregularidades em corrupção e má gestão. As irregularidades classificadas como "licitação" serão proxy para corrupção, já que envolvem fraudes e irregularidades em licitações, a forma principal de trocas entre gestores públicos e atores do mercado. As demais irregularidades relacionadas a "gastos", "execução" e "utilização" de programas públicos serão proxy para má gestão. Exemplos do tópico "licitação" são a irregularidade "Montagem de processo licitatório", "Direcionamento de licitação para construção de quadras poliesportivas e reforma de escola para contratação de empresa não localizada no endereço informado" e "Subcontratação total do contrato de transporte escolar de no 0122.01/2013.01, o que gerou, nos Exercícios 2013 e 2014, um sobrepreço no montante de R\$ 120.525,00 com recursos do Fundeb”. Essas irregularidades se aproximam mais da definição de corrupção, uma vez que indicam desvios de verbas. 
Os demais tópicos envolvem irregularidades menores, como a irregularidade "Aplicação de recursos do Índice de Gestão Descentralizada (IGD) em despesas não elegíveis para o Programa Bolsa Família”, classificada como "gastos"; a irregularidade "Órgão de Controle Social do Programa Bolsa Família não vem exercendo de forma eficiente suas funções", classificada como "execução"; e a irregularidade "Utilização de veículos inadequados para o transporte de alunos", classificada como "utilização".

A partir da agregação das irregularidades, temos informações sobre a contagem de irregularidades totais, de corrupção e de má gestão. Contudo, apesar de ser o procedimento comum nas análises que usam os dados da CGU, a simples contagem de irregularidades pode ser uma medida enviesada, já que em diferentes municípios o número de programas auditados pode ser diferente e isso afeta diretamente o número absoluto de irregularidades identificadas. Para solucionar esse problema e apresentar medidas mais válidas, ponderamos o número absoluto de irregularidades totais, corrupção e má gestão pelo número de programas auditados no município. Essa informação consta na "ordem de serviço" da auditoria da CGU e os dados foram disponibilizados por Gehrke (2019).

As variáveis finais são, portanto, o número de irregularidades ponderado pelo número de objetos/ programas auditados como informado na ordem de serviço. A primeira especificação é quanto ao número total de irregularidades no município. A segunda especificação é o número de irregularidades classificadas como má gestão. A terceira especificação é o número de irregularidades classificadas como corrupção. Como as variáveis dependentes do estudo são todas contínuas, foram usados modelos de regressão linear por mínimos quadrados ordinários.

Como variáveis de controle incluímos as variáveis reeleição, qualidade da burocracia, meios de comunicação, desenvolvimento econômico e região. Reeleição assume valor 0 quando o prefeito está no primeiro mandato e valor 1 quando está no segundo. Qualidade da burocracia indica o percentual de funcionários da burocracia local com Ensino Superior. Meios de comunicação indica o número de veículos disponíveis no município. As opções são rádio comunitária, rádio $\mathrm{AM}$, rádio $\mathrm{FM}$, internet, televisão. Então, a variável assume o valor mínimo 0 para municípios sem meios de comunicação e 5 para municípios com todos os meios. Como medida de desenvolvimento econômico usamos o PIB per capita do município. Por último, incluímos a região do país como conjunto de variáveis binárias. Todos os dados são do IBGE.

A crescente preocupação com inferências causais válidas fez os cientistas políticos buscarem formas de identificar o contrafactual, ou o que aconteceria se a variável de interesse não ocorresse. $\mathrm{O}$ uso de modelos de regressão para essa empreitada sofre de uma série de limitações, já que, mesmo com a inclusão de variáveis de controle para identificar a relação entre X e Y e manter esses fatores constantes, o problema de variável omitida ainda está presente, o que pode ocasionar problemas de endogeneidade. A estratégia aqui adotada foi o uso de controles apontados como relevantes pela literatura e a cautela na interpretação dos resultados, já que não foram produzidos a partir de um experimento controlado ${ }^{3}$.

\footnotetext{
${ }^{3} \mathrm{O}$ uso das chamadas técnicas quase experimentais é uma saída estatística para situações onde um experimento controlado não é viável. Algumas dessas técnicas poderiam ser consideradas neste estudo. Contudo, os dados da CGU que revelam as irregularidades nos municípios só existem para 320 municípios. Dessa forma, técnicas como pareamento ou regressão descontínua tem pouca aplicabilidade, já que poucos casos sobrariam após um procedimento de pareamento ou o uso de regressão descontínua com eleições apertadas como variável de quebra. Além disso, essas técnicas também têm suas próprias limitações, que devem ser consideradas.
} 


\section{RESULTADOS}

Como argumentamos anteriormente, para a efetiva implementação da LAI (2011), os municípios precisam regulamentá-la em nível local, estabelecendo procedimentos próprios para o acesso à informação pública. A Figura 2 apresenta o número e o ritmo de regulamentação da LAI (2011) pelos municípios brasileiros. No painel à esquerda são apresentados todos os municípios do Brasil e no painel à direita temos os municípios incluídos na nossa amostra de localidades auditadas pela CGU.

\section{FIGURA 2 ADOÇÃO DA LEI DE ACESSO À INFORMAÇÃO PELOS MUNICÍPIOS BRASILEIROS}

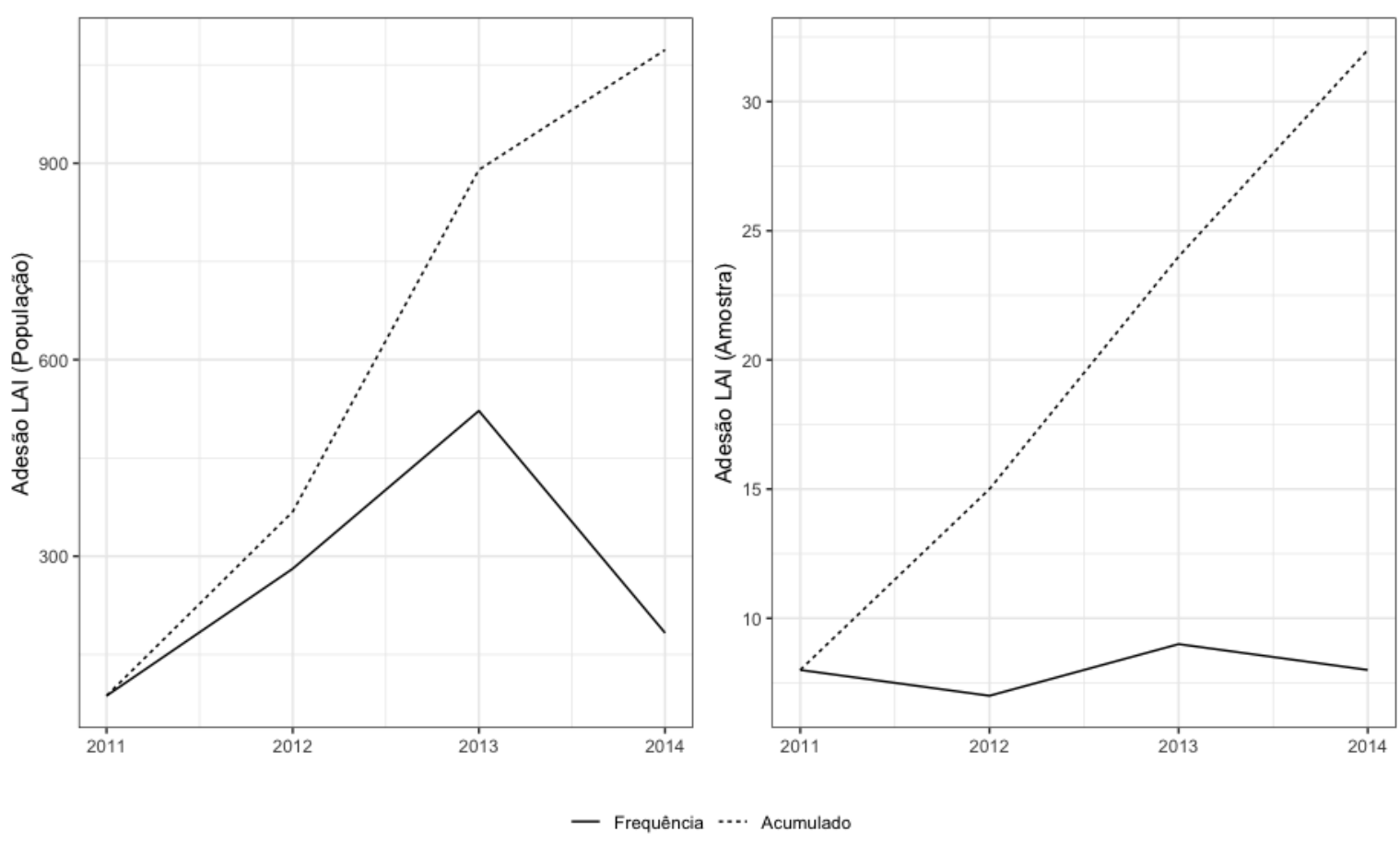

Fonte: Elaborada pelos autores.

A Figura 2 mostra que o número e o ritmo de regulamentação da LAI (2011) podem ser considerados baixo e lento, respectivamente. $\mathrm{O}$ número de adotantes cresceu, atingindo o número máximo em 2013. Contudo, esse crescimento não se manteve linear, com redução do número de adotantes em 2014. Considerando o número acumulado, apenas 1 em cada 5 municípios do Brasil regulamentaram a LAI (2011). A amostra de municípios auditados pela CGU inclui apenas municípios menores. A distribuição é semelhante à da população, mas os números são bem menores. Enquanto $20 \%$ dos municípios brasileiros regulamentaram a LAI (2011), na amostra, esse valor cai para 10\%. Isso ocorre porque os municípios com maior chance de regulamentação são justamente os maiores e as capitais (Batista, 2017). Tendo em mente que a ausência de regulamentação limita consideravelmente o acesso à informação, uma vez que os procedimentos não estão formalizados, podemos concluir que a transparência em nível local ainda é muito limitada. 
A Figura 2 mostra que, após 4 anos da instituição da LAI (2011) no Brasil, a maior parte dos municípios ainda não regulamentou a lei em nível local. Mais importante, o número de adotantes diminuiu em 2014, indicando que não há tendência de crescimento linear na regulamentação da LAI (2011). Por si, esse resultado já indica que a LAI (2011) nacional não se traduz automaticamente no compromisso institucional com a transparência em nível local e também que maiores investimentos precisarão ser realizados para que os municípios se adequem a nova realidade de transparência pública. Além disso, dos municípios que adotaram, a maioria adotou em 2013, indicando que ainda há pouca experiência com a lei e, por isso, menor institucionalização dos procedimentos. A Tabela 1 mostra os resultados quanto ao grau de regulamentação em nível local.

\section{TABELA 1 GRAU DE REGULAMENTAÇÃO DA LAI NOS MUNICÍPIOS BRASILEIROS}

\begin{tabular}{|c|c|c|c|}
\hline & Mín. & Máx. & Média \\
\hline População (total) & 0 & 8,00 & 0,818 \\
\hline $\begin{array}{l}\text { População } \\
\text { (adotantes) }\end{array}$ & 1,00 & 8,00 & 4,239 \\
\hline Amostra (total) & 1,00 & 8,00 & 0,684 \\
\hline Amostra (adotantes) & 1,00 & 8,00 & 4,576 \\
\hline
\end{tabular}

Fonte: El aborada pelos autores.

O propósito de incluir essa variável é ter uma medida mais refinada dos procedimentos para a transparência pública nos municípios. A Tabela 1 indica que o grau de regulamentação ainda é baixo, principalmente quando consideramos todos os municípios. A média de procedimentos quando consideramos os municípios que adotaram e os que não adotaram é de 0,818 para a população e 0,684 para a amostra. Como o valor máximo desse índice é 8 , mostramos que a formalização de procedimentos é extremamente baixa. Quando consideramos apenas os municípios que regulamentaram, a média é de 4,239 para a população e de 4,576 para a amostra. Dessa forma, mesmo considerando apenas os municípios que aderiram à LAI (2011), a formalização dos procedimentos se encontra longe do ideal.

Os resultados sobre a adesão à LAI (2011) pelos municípios brasileiros mostram que ainda há muito a ser feito em nível local. A instituição nacional da LAI (2011) não implica automaticamente a formulação de procedimentos que autorizem e incentivem o acesso à informação pública. Se, por um lado, a difusão da transparência em nível local é limitada, como está distribuída a performance dos governos locais? 

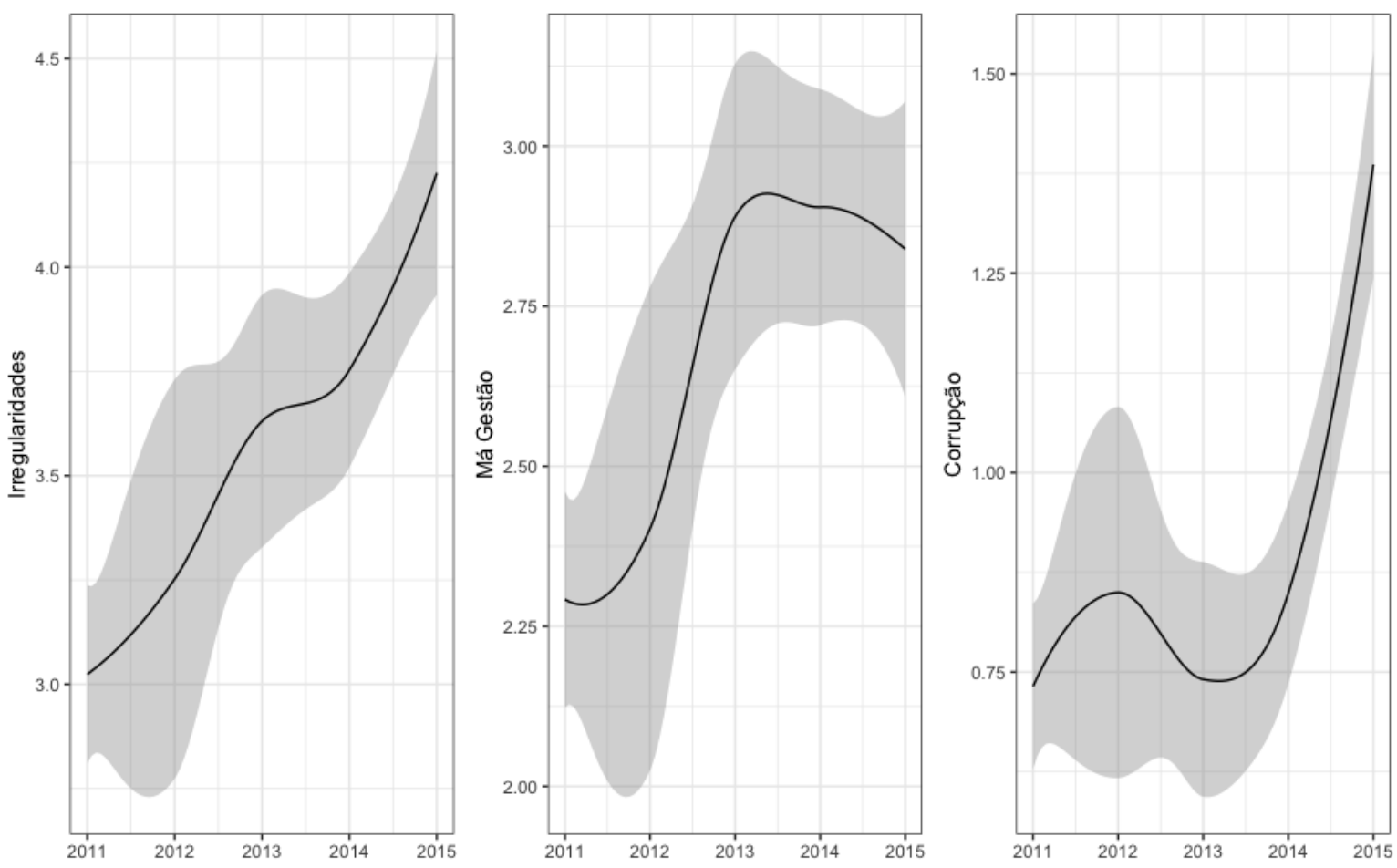

Fonte: Elaborada pelos autores.

Os 3 painéis da Figura 3 mostram a distribuição de irregularidades observadas nos municípios auditados pela CGU. Os valores absolutos das irregularidades são ponderados pelo número de programas auditados no município. A média é de 3,5 irregularidades por projeto auditado, mostrando um número significativo de problemas na gestão de recursos federais pelos governos locais. Para má gestão, a média é de 2,6 casos por projeto. A média de casos de corrupção é menor, com 0,88 casos por projeto auditado. Dessa forma, dentre as irregularidades observadas, a má gestão é a mais presente e a corrupção é observada em menor grau. Em todos os casos, contudo, os números são expressivos, indicando que é necessária intervenção para reduzir esses problemas na entrega de benefícios e serviços públicos.

Agora, o objetivo passa a ser identificar se os municípios que regulamentaram a LAI (2011) apresentam alguma melhora em sua performance. Dito de outra forma, o compromisso institucional com a transparência tem relação negativa com a corrupção e a má gestão? A Tabela 2 apresenta os resultados dos modelos de regressão que identificam a associação entre a transparência e a performance, controlando pelos fatores que também podem afetar o desempenho do governo. 
RAP | Transparência, corrupção e má gestão: uma análise dos municípios brasileiros

\section{TABELA 2 TRANSPARÊNCIA E IRREGULARIDADES NA GESTÃO MUNICIPAL}

\begin{tabular}{|c|c|c|c|c|c|c|c|c|c|}
\hline & \multicolumn{9}{|c|}{ Variável dependente } \\
\hline & \multicolumn{3}{|c|}{ Irregularidades } & \multicolumn{3}{|c|}{ Má Gestão } & \multicolumn{3}{|c|}{ Corrupção } \\
\hline & (1) & (2) & (3) & (4) & (5) & (6) & (7) & (8) & (9) \\
\hline \multirow[t]{2}{*}{ Regulamentação } & 0,123 & & & 0,114 & & & 0,009 & & \\
\hline & $(0,224)$ & & & $(0,186)$ & & & $(0,114)$ & & \\
\hline \multirow[t]{2}{*}{ Grau } & & 0,057 & & & $0,052^{*}$ & & & 0,005 & \\
\hline & & $(0,036)$ & & & $(0,030)$ & & & $(0,018)$ & \\
\hline \multirow[t]{2}{*}{ Institucionalização } & & & 0,037 & & & $-0,004$ & & & 0,041 \\
\hline & & & $(0,080)$ & & & $(0,067)$ & & & $(0,041)$ \\
\hline \multirow{2}{*}{$\begin{array}{l}\text { Meios de } \\
\text { comunicação }\end{array}$} & $-0,036$ & $-0,038$ & $-0,034$ & $-0,057$ & $-0,059$ & $-0,053$ & 0,021 & 0,021 & 0,020 \\
\hline & $(0,045)$ & $(0,045)$ & $(0,045)$ & $(0,038)$ & $(0,037)$ & $(0,038)$ & $(0,023)$ & $(0,023)$ & $(0,023)$ \\
\hline \multirow[t]{2}{*}{ Burocracia } & $-0,016^{* *}$ & $-0,016^{* *}$ & $-0,016^{* *}$ & $-0,009$ & $-0,010^{*}$ & $-0,009$ & $-0,007^{* *}$ & $-0,007^{* *}$ & $-0,007^{* *}$ \\
\hline & $(0,006)$ & $(0,006)$ & $(0,006)$ & $(0,005)$ & $(0,005)$ & $(0,005)$ & $(0,003)$ & $(0,003)$ & $(0,003)$ \\
\hline \multirow[t]{2}{*}{ Reeleito } & 0,089 & 0,086 & 0,092 & $-0,002$ & $-0,004$ & 0,003 & 0,091 & 0,090 & 0,089 \\
\hline & $(0,137)$ & $(0,136)$ & $(0,138)$ & $(0,114)$ & $(0,113)$ & $(0,115)$ & $(0,070)$ & $(0,070)$ & $(0,070)$ \\
\hline \multirow{2}{*}{ PIB per capita } & $0,003^{*}$ & $0,003^{* *}$ & $0,003^{*}$ & 0,002 & $0,003^{* *}$ & $0,003^{* *}$ & 0,0005 & 0,001 & 0,0003 \\
\hline & $(0,001)$ & $(0,001)$ & $(0,002)$ & $(0,001)$ & $(0,001)$ & $(0,001)$ & $(0,001)$ & $(0,001)$ & $(0,001)$ \\
\hline \multirow[t]{2}{*}{ Ano } & $0,277^{*+*}$ & $0,279^{\cdots \cdots}$ & $0,277^{* \star * x}$ & $0,163^{m *}$ & $0,166^{* * *}$ & $0,168^{+* * *}$ & $0,114^{m * *}$ & $0,114^{* * * *}$ & $0,109^{* * * *}$ \\
\hline & $(0,043)$ & $(0,042)$ & $(0,043)$ & $(0,035)$ & $(0,035)$ & $(0,036)$ & $(0,022)$ & $(0,021)$ & $(0,022)$ \\
\hline \multirow[t]{2}{*}{ Nordeste } & $0,807^{\cdots *}$ & 0,832 & $0,801^{\cdots \cdots}$ & $0,713^{\cdots \cdots}$ & $0,735^{\ldots *}$ & $0,706^{* \cdots}$ & 0,095 & 0,097 & 0,095 \\
\hline & $(0,249)$ & $(0,248)$ & $(0,249)$ & $(0,207)$ & $(0,206)$ & $(0,207)$ & $(0,127)$ & $(0,127)$ & $(0,127)$ \\
\hline \multirow[t]{2}{*}{ Norte } & $0,822^{* * *}$ & $0,853^{\cdots *}$ & $0,819^{m * *}$ & $0,800^{\cdots * *}$ & $0,828^{* * * *}$ & $0,792^{* * *}$ & 0,022 & 0,025 & 0,027 \\
\hline & $(0,300)$ & $(0,300)$ & $(0,301)$ & $(0,250)$ & $(0,249)$ & $(0,250)$ & $(0,153)$ & $(0,153)$ & $(0,153)$ \\
\hline
\end{tabular}




\begin{tabular}{|c|c|c|c|c|c|c|c|c|c|}
\hline & \multicolumn{9}{|c|}{ Variável dependente } \\
\hline & \multicolumn{3}{|c|}{ Irregularidades } & \multicolumn{3}{|c|}{ Má Gestão } & \multicolumn{3}{|c|}{ Corrupção } \\
\hline & (1) & (2) & (3) & (4) & (5) & (6) & (7) & (8) & (9) \\
\hline \multirow[t]{2}{*}{ Sudeste } & $-0,156$ & $-0,147$ & $-0,160$ & 0,202 & 0,209 & 0,191 & $-0,357^{4+*+}$ & $-0,356^{* * *}$ & $-0,352^{+* * *}$ \\
\hline & $(0,257)$ & $(0,256)$ & $(0,257)$ & $(0,214)$ & $(0,212)$ & $(0,214)$ & $(0,131)$ & $(0,131)$ & $(0,131)$ \\
\hline \multirow[t]{2}{*}{ Sul } & $-0,178$ & $-0,189$ & $-0,180$ & 0,140 & 0,130 & 0,148 & $-0,318^{* *}$ & $-0,319^{* *}$ & $-0,328^{* *}$ \\
\hline & $(0,275)$ & $(0,274)$ & $(0,276)$ & $(0,229)$ & $(0,228)$ & $(0,230)$ & $(0,140)$ & $(0,140)$ & $(0,140)$ \\
\hline \multirow[t]{2}{*}{ Constante } & $-554,101^{+* *+x}$ & $-558,747^{\ldots *}$ & $-555,043^{* * * x}$ & $-326,594^{\ldots * *}$ & $-330,970^{* * * *}$ & $-336,533^{* * *}$ & $-227,507^{* * * x}$ & $-227,776$ & $-218,510^{*+* x}$ \\
\hline & $(85,635)$ & $(83,679)$ & $(85,994)$ & $(71,221)$ & $(69,539)$ & $(71,557)$ & $(43,625)$ & $(42,791)$ & $(43,712)$ \\
\hline Observações & 285 & 285 & 284 & 285 & 285 & 284 & 285 & 285 & 284 \\
\hline $\mathrm{R}^{2}$ & 0,278 & 0,284 & 0,276 & 0,183 & 0,191 & 0,181 & 0,203 & 0,203 & 0,203 \\
\hline $\mathrm{R}^{2}$ ajustado & 0,252 & 0,257 & 0,249 & 0,153 & 0,161 & 0,151 & 0,173 & 0,174 & 0,174 \\
\hline Doidual Ctd $\mathrm{C}$ & 1,086 & 1,082 & 1,088 & 0,903 & 0,899 & 0,905 & 0,553 & 0,553 & 0,553 \\
\hline Resiaual sta. Error & $(\mathrm{df}=274)$ & $(\mathrm{df}=274)$ & $(\mathrm{df}=273)$ & $(\mathrm{df}=274)$ & $(\mathrm{df}=274)$ & $(\mathrm{df}=273)$ & $(\mathrm{df}=274)$ & $(\mathrm{df}=274)$ & $(\mathrm{df}=273)$ \\
\hline $\mathrm{F}$ & $10,545^{\text {tata }}$ & $10,843^{+*+*}$ & $10,384^{*+*+}$ & $6,148^{\cdots+*}$ & $6,466^{*+*}$ & $6,040^{\cdots+\cdots}$ & $6,958^{4+*+}$ & $6,966^{*+*}$ & $6,973^{m+*}$ \\
\hline$r$ & $(\mathrm{df}=10 ; 274)$ & $(\mathrm{df}=10 ; 274)$ & $(\mathrm{df}=10 ; 273)$ & $(\mathrm{df}=10 ; 274)$ & $(\mathrm{df}=10 ; 274)$ & $(\mathrm{df}=10 ; 273)$ & $(\mathrm{df}=10 ; 274)$ & $(\mathrm{df}=10 ; 274)$ & $(\mathrm{df}=10 ; 273)$ \\
\hline
\end{tabular}

Nota: ${ }^{*} \mathrm{p}^{* *} \mathrm{p}^{\star * *} \mathrm{p}<0,01$

Fonte: Elaborada pelos autores.

Na Tabela 2, apresentamos 3 modelos diferentes para cada especificação da variável dependente. Dessa forma, apresentamos as 3 medidas de transparência propostas neste estudo. A primeira é a regulamentação da LAI (2011), a segunda é o grau de regulamentação e a terceira é o tempo de adesão como medida de institucionalização da LAI (2011) em nível local. Os resultados indicam que em todas as especificações da variável dependente que buscam captar a performance governamental, as medidas de transparência não apresentam associação estatisticamente significativa. Esse resultado indica que, mantendo os demais fatores constantes, a transparência não diminui a ocorrência de irregularidades na gestão local, não diminui a má gestão ou a corrupção.

A literatura que busca identificar empiricamente o efeito da transparência no desempenho do governo encontra resultados mistos. Alguns mostram o efeito positivo da transparência para melhorar o desempenho do governo, principalmente diminuindo a corrupção, seu objetivo maior. Outros estudos, no entanto, não encontram efeito estatisticamente significativo. Contribuindo para essa literatura, mostramos que, no caso dos municípios brasileiros, o compromisso institucional com a transparência não se mostra um fator forte o suficiente para reduzir as irregularidades na gestão local, melhorando o desempenho do governo. Tendo em vista que a maior justificativa para a difusão de iniciativas de transparência é a qualidade do governo, os resultados nulos contribuem 
para a literatura, que é notadamente dividida entre os resultados empíricos alcançados e também as iniciativas de transparência, que precisam ir além do compromisso institucional ${ }^{4}$.

Do ponto de vista do desenho de pesquisa, uma questão seria a possibilidade de endogeneidade na relação entre transparência e performance governamental. Isso porque o tratamento, a adesão à LAI (2011), não é distribuído aleatoriamente. Isto é, os municípios escolhem regulamentar a LAI (2011), o momento da adesão e também o grau de regulamentação. Dessa forma, municípios com melhores resultados poderiam escolher aderir à LAI (2011) por não terem “nada a esconder", e isso caracterizaria uma relação endógena. Contudo, se houvesse endogeneidade, o que não podemos descartar com o desenho de pesquisa aqui implementado, e ela de fato afetasse os nossos resultados, as estimativas estariam na direção contrária. Isto é, seria mais provável encontrar a associação negativa entre transparência e performance, já que os municípios com melhor performance teriam mais incentivos para aderir à LAI (2011), de modo que a performance anterior causaria tanto a transparência (VI) quanto a performance (VD). Dado que os nossos resultados indicam que a transparência não tem relação com a performance governamental, temos indícios de que a endogeneidade não é determinante dos resultados aqui apresentados. Em resumo, no estado atual de difusão da LAI (2011) nos municípios, a regulamentação, o grau de regulamentação ou o tempo de adesão não apresentam relação com a performance governamental.

Sobre as variáveis que apresentam resultado estatisticamente significativo, encontramos que a qualidade da burocracia diminui o número de irregularidades, má gestão e corrupção. Ou seja, quanto maior a escolaridade dos funcionários na administração local, menor o número de irregularidades identificadas. Esse resultado é condizente com pesquisas anteriores, que mostram o efeito da qualidade da burocracia local sobre a performance dos municípios (Batista, 2015; Marenco, 2017; Marenco et al., 2017). Dessa forma, uma burocracia de qualidade tem efeito significativo para diminuir as irregularidades cometidas pelos gestores e, assim, aumentar a entrega de benefícios e serviços à população.

Outra variável que apresentou resultado estatisticamente significativo foi o desenvolvimento econômico. Os resultados indicam que em todas as especificações, com exceção de corrupção, quanto maior o PIB per capita local, maior o número de irregularidades observadas. Esse resultado indica que em municípios maiores e, consequentemente, mais complexos, que administram mais recursos, observa-se maior número de irregularidades e de casos de má gestão. $\mathrm{O}$ conjunto de variáveis que indicam a região do município, que têm a região Centro-Oeste como categoria de referência, indica que as regiões Norte e Nordeste geralmente exibem maior número de irregularidades totais e de casos de má gestão. Nos casos de corrupção, as regiões Sul e Sudeste apresentam menos casos do que a região Centro-Oeste. Por último, a variável ano também apresenta resultados estatisticamente significativos, mostrando que o número de irregularidades está crescendo no tempo e não vem diminuindo, como se poderia esperar, dada a implementação da LAI (2011) em âmbito nacional.

\footnotetext{
${ }^{4}$ Ressaltamos a importância da divulgação de resultados nulos, especialmente na área de políticas públicas e intervenção governamental. Isso porque a omissão de resultados que não são estatisticamente significativos caracteriza viés de publicação e também incentiva a manutenção de iniciativas que, muitas vezes, não geram os resultados esperados.
} 


\section{CONCLUSÃO}

O compromisso institucional com a transparência pública diminui a corrupção e a má gestão governamental? Essa foi a pergunta que procuramos responder neste estudo. Com base no argumento geral de que os governantes se comportam melhor quando são observados, existe uma expectativa teórica e prática de que transparência reduza corrupção e melhore a performance do governo. Todavia, as evidências sobre os efeitos das políticas de transparência ao redor do mundo são heterogêneas, apontando diferentes resultados.

Nesse sentido, visamos a contribuir com esse debate usando uma medida objetiva de corrupção e má gestão a partir de um banco original, que compila cerca de 16 mil irregularidades identificadas pela CGU no período de 2011 a 2015. Testamos as hipóteses de que a regulamentação, o grau de regulamentação e a institucionalização da LAI (2011) estão associados a menor nível de irregularidades de forma geral, de corrupção e de má gestão nos municípios brasileiros.

Nossos resultados indicam que a transparência, nas três especificações usadas, não diminui a ocorrência de irregularidades na gestão local, nem reduz a má gestão e a corrupção. Qualidade da burocracia e desenvolvimento econômico, por sua vez, apresentaram resultados estatisticamente significativos e na direção esperada. Com isso, contribuímos para o debate ao trazer evidências da relação entre o compromisso institucional e a transparência sobre medidas diretas e objetivas de corrupção e má gestão como proxies de performance do governo.

Isso não significa que a LAI (2011), ou a transparência no governo, de forma mais ampla, não importa para o combate à corrupção e para a melhoria da gestão pública. Nosso desenho de pesquisa é quantitativo e probabilístico. Uma vez identificados esses resultados, futuros estudos podem avançar na agenda de pesquisa dos mecanismos causais na relação entre transparência e performance, seja desenvolvendo medidas de transparência efetiva, seja identificando em qual elo da cadeia causal o compromisso institucional com a transparência perde sua capacidade de afetar a performance governamental. Para esse ponto serão necessários desenhos baseados em process-tracing ou que identifiquem claramente o contrafactual ${ }^{5}$.

Ademais, como enfatiza Michener (2019), os efeitos das políticas de transparência tendem a ser graduais, indiretos e difusos. Nesse sentido, nossos resultados indicam que ainda há um longo caminho pela frente em termos de fortalecimento da LAI (2011) em nível subnacional. Antes, é preciso garantir a efetiva implementação da lei e dos mecanismos de acesso à informação, para que possamos, de fato, visualizar seus efeitos na sociedade. Assim, sugerimos como agenda de pesquisa o aprofundamento da análise sobre o grau de regulamentação e institucionalização da LAI (2011) nos municípios brasileiros.

\footnotetext{
${ }^{5} \mathrm{O}$ contrafactual é a identificação do que aconteceria caso X, a variável de interesse, não ocorresse. É a forma de isolar o efeito causal de $\mathrm{X}$ sobre Y. A busca pelo contrafactual é bastante discutida em desenhos de pesquisa quantitativos e experimentais. Contudo, também é possível buscar o contrafactual em desenhos qualitativos, que são orientados para casos e não variáveis. Uma forma de buscar o contrafactual é por meio de "experimentos mentais" (Mitchell, 2004). Outra forma de analisar relações causais em desenhos qualitativos e orientados para casos é o chamado process-tracing - que tem como foco a análise de mecanismos causais. A proposta é abrir a "caixapreta" da causalidade usando métodos de estudo de caso em profundidade (Beach \& Pedersen, 2019).
} 


\section{REFERÊNCIAS}

Adserà, A., Boix, C., \& Payne, M. (2003). Are you being served? Political accountability and quality of government. Journal of Law, Economics, \& Organization, 19(2), 445-490.

Albuquerque, B. E., \& Ramos, F. S. (2006). Análise teórica e empírica dos determinantes de corrupção na gestão pública municipal. In Anais do 340 Encontro Nacional de Economia. Salvador, BA.

Andrews, R., Boyne, G. A., \& Enticott, G. (2006). Performance failure in the public sector. Public Management Review, 8(2), 273-296.

Barro, R. J. (1973). The control of politicians: an economic model. Public Choice, 14, 19-42.

Bataglia, M. B. (2019). Acesso à informação e corrupção: investigando o contexto institucional da CGU (Dissertação de Mestrado). Universidade de Brasília, Brasília, DF.

Batista, M. (2013). Incentivos da dinâmica política sobre a corrupção: reeleição, competitividade e coalizões nos municípios brasileiros. Revista Brasileira de Ciências Sociais, 28(82), 87-106.

Batista, M. (2015). Burocracia local e qualidade da implementação de políticas públicas descentralizadas: uma análise da gestão de recursos federais pelos municípios brasileiros. Revista do Serviço Público, 66(3), 345-370.

Batista, M. (2017). A difusão da lei de acesso à informação nos municípios brasileiros: fatores internos e externos. Brasília, DF: Escola Nacional de Administração Pública.

Bauhr, M., \& Grimes, M. (2012, December). What is government transparency? New measures and relevance for quality of government (Working Paper Series 2012). Gothenburg, Sweden: The Quality of Government Institute.

Beach, D., \& Pedersen, R. B. (2019). Process-tracing methods: foundations and guidelines. Ann Arbor, MI: University of Michigan Press.

Bentham, J. (1995). The panopticon writings. London, UK: Verso.

Berliner, D. (2014). The political origins of transparency. The Journal of Politics, 76(2), 479-491.
Blei, D. (2012). Probabilistic topic models. Communications of the ACM, 5(4), 77-84.

Brunetti, A., \& Weder, B. (2003). A free press is bad news for corruption. Journal of Public Economy, 87(7-8), 1801-1824.

Calland, R., \& Bentley, K. (2013). The impact and effectiveness of transparency and accountability initiatives: freedom of information. Development Policy Review, 31, 69-87.

Casalecchi, A. R. C., \& Oliveira, E. M. (2010). As auditorias da CGU e a transparência licitatória dos municípios paulistas. Cadernos Gestão Pública e Cidadania, 15, 49-62.

Chang, E. C. C. (2005). Electoral incentives for political corruption under open-list proportional representation. The Journal of Politics, 67(3), 716730.

Chang, E. C. C., \& Golden, M. A. (2007). Electoral systems, district magnitude and corruption. British Journal of Political Science, 37(1), 115-137.

Controladoria-Geral da União. (2013). Manual da Lei de Acesso à Informação para estados e municípios. Brasília, Autor.

Costa, S. (2012). Do freedom of information laws decrease corruption? The Journal of Law, Economics, and Organization, 29(6), 1317-1343.

Cucciniello, M., Grimmelikhuijsen, S., \& Porumbescu, G. A. (2016). 25 years of transparency research: evidence and future directions. Public Administration Review, 77(1), 32-44.

Escaleras, M., Lin, S., \& Register, C. (2010). Freedom of information acts and public sector corruption. Public Choice, 145(3), 435-460.

Ferejohn, J. (1986). Incumbent performance and electoral control. Public Choice, 50(1-3), 5-25.

Ferraz, C., \& Finan, F. (2011). Electoral accountability and corruption: evidence from the audit reports of local governments. American Economic Review, 101(4), 1274-1311.

Fox, J. (2007). The uncertain relationship between transparency and accountability. Development in Practice, 17(4-5), 663-671.

Freedom of Information. (2019). Alphabetical and chronological lists of countries with FOI 
regimes. Recuperado de http://www.freedominfo. org/?p=18223

Fukuyama, F. (2013). What is governance? Governance: An International Journal of Policy, Administration, and Institutions, 26(3), 347-386.

Fukuyama, F. (2016). Governance: what do we know, and how do we know it. Annual Review of Political Science, 19, 89-105.

Gehrke, M. (2019). How politicians react to anticorruption investigations and enforcement. Evidence from Brazilian municipalities (Ph.D. Dissertation). University of California in Los Angeles, Los Angeles, CA.

Heald, D. (2006). Varieties of transparency. In C. Hood, \& D. Heald (Org.), Transparency: the key to better governance? (pp. 25-43). Oxford, UK: Oxford University Press.

Hollyer, J. R., Rosendorff, P. B. \& Vreeland, J. R. (2014). Measuring transparency. Political Analysis, $22,413-434$.

Instituto Brasileiro de Geografia e Estatistica. (2020). Pesquisa de Informações Básicas Municipais - MUNIC. Recuperado de https://www.ibge.gov.br/ estatisticas/sociais/educacao/10586-pesquisa-deinformacoes-basicas-municipais.html $=\& \mathrm{t}=0$-que-e

Jain, A. K. (2002). Corruption: a review. Journal of Economic Surveys, 15(1), 71-121.

Kosack, S., \& Fung, A. (2014). Does transparency improve governance? Annual Review of Political Science, 17, 65-87.

Lei n. 12.527, de 18 de novembro de 2011. (2011). Regula o acesso a informações previsto no inciso XXXIII do art. $5^{\circ}$, no inciso II do $\$ 3^{\circ}$ do art. 37 e no $\$ 2^{\circ}$ do art. 216 da Constituição Federal; altera a Lei n. 8.112, de 11 de dezembro de 1990; revoga a Lei n. 11.111, de 5 de maio de 2005, e dispositivos da Lei n. 8.159, de 8 de janeiro de 1991; e dá outras providências. Brasília, DF.

Lindstedt, C., \& Naurin, D. (2010). Transparency is not enough: making transparency effective in reducing corruption. International Political Science Review, 31(3), 301-322.

Marenco, A. (2017). Burocracias profissionais ampliam capacidade estatal para implementar políticas? Governos, burocratas e legislação em municípios brasileiros. Dados, 60(4), 1025-1058.
Marenco, A., Strohschoen, M. T. B., \& Joner, W. (2017). Capacidade estatal, burocracia e tributação nos municípios brasileiros. Revista de Sociologia e Política, 25(64), 3-21.

Meijer, A., Curtin, D., \& Hillebrandt, M. (2012). Open government: connecting vision and voice. International Review of Administrative Sciences, 78(1), 10-29.

Michener, G. (2019). Gauging the impact of transparency policies. Public Administration Review, 79(1), 136-139.

Michener, G., \& Bersch, K. (2013). Identifying transparency. Information Polity,18(3), 233-242.

Mitchell, G. (2004). Case studies, counterfactuals, and causal explanations. University of Pennsylvania Law Review, 152(5), 1517-1608.

Nyblade, B., \& Reed, S. R. (2008). Who cheats? Who loots? Political competition and corruption in Japan, 1947-1993. American Journal of Political Science, 52(4), 926-941.

Pereira, C, Melo, M. A., \& Figueiredo, C. M. (2008). The corruption-enhancing role of re-elections incentives? Counterintuitive evidence from Brazil's audit reports. Political Research Quaterly, 62(4), 731-744.

Persson, T., Tabellini, G., \& Trebbi, F. (2003). Electoral rules and corruption. Journal of the European Economic Association, 1, 958-989.

Prat, A. (2005). The wrong kind of transparency. The American Economic Review, 95(3), 862-877.

Rodrigues, K. F. (2019). Desvelando o conceito de transparência: seus limites, suas variedades e a criação de uma tipologia. Cadernos EBAPE.BR, 18(2), 237-253.

Rothstein, B., \& Teorell, J. (2008). What is quality of government? A theory of impartial government institutions. Governance: An International Journal of Policy, Administration, and Institutions, 21(2), 165-190.

Schedler, A. (1999). Conceptualizing accountability. In A. Schedler, L. Dimond, \& M. F. Plattner (Ed.), The self-restraining State. London, UK: Lynne Rienner.

Schnell, S. (2017). Cheap talk or incredible commitment? (Mis)calculating transparency and anti-corruption. Governance, 31(3), 415-430. 
RAP | Transparência, corrupção e má gestão: uma análise dos municípios brasileiros

Shleifer, A., \& Vishny, R. W. (1993). Corruption. The Quarterly Journal of Economics, 108(3), 599-617.

Sodré, A. C. A., \& Alves, M. F. C. (2010). Relação entre emendas parlamentares e corrupção municipal no Brasil: estudo dos relatórios do programa de fiscalização da Controladoria-Geral da União. Revista de Administração Contemporânea, 14(3), 414-433.

\section{Mariana Batista}

https://orcid.org/0000-0002-2456-4164

Doutora em Ciência Política; Professora adjunta do Departamento de Ciência Política da Universidade Federal de Pernambuco (UFPE). E-mail: mariana.bsilva@gmail.com

\section{Virginia Rocha}

iD

https://orcid.org/0000-0001-5267-4929

Doutoranda em Ciência Política no Programa de Pós-Graduação em Ciência Política da Universidade Federal de Pernambuco (UFPE). E-mail: vii.rrocha@gmail.com

\section{José Luiz Alves dos Santos}

https://orcid.org/0000-0003-4387-3410

Graduando em Ciência Política da Universidade Federal de Pernambuco(UFPE).E-mail: jsluizz18@gmail.com 